Research Article

\title{
New Simplified Method for Parameter Estimation of Three-Dimensional Channel Model in Third Generation Partnership Project
}

\author{
Hanene Zormati $\mathbb{D}^{1},{ }^{1}$ Jalel Chebil, $^{2}$ and Jamel Belhadj Taher ${ }^{1}$ \\ ${ }^{1}$ University of Sousse, National Engineering School of Sousse, NOCCS Laboratory, Sousse, Tunisia \\ ${ }^{2}$ University of Sousse, Higher Institute of Transport and Logistics of Sousse, NOCCS Laboratory, Sousse, Tunisia
}

Correspondence should be addressed to Hanene Zormati; zormati.hanen@hotmail.fr

Received 21 January 2021; Revised 18 May 2021; Accepted 24 June 2021; Published 3 July 2021

Academic Editor: Ana Alejos

Copyright (c) 2021 Hanene Zormati et al. This is an open access article distributed under the Creative Commons Attribution License, which permits unrestricted use, distribution, and reproduction in any medium, provided the original work is properly cited.

\begin{abstract}
The emerging fifth generation technology has attracted the attention of many researchers and developers. It is designed to connect everyone and everything together including machines, vehicles, objects, and devices. One of the fifth generation research topics that are being investigated is the modelling of the wireless communication channel. In this paper, Third Generation Partnership Project channel models are studied and its variant Three-Dimensional Third Generation Partnership Project model is examined. This model is complex and not easy to use due to the employment of a large number of parameters. For these reasons, a new simplified method for parameter estimation of the three-dimensional model of third generation partnership project based on estimation of signal parameters via rotational invariant techniques algorithm is proposed. The simplified model is less complex than the three-dimensional model of third generation partnership project and is found to perform better in terms of computational time while maintaining similar results.
\end{abstract}

\section{Introduction}

The fifth generation (5G) systems will facilitate the exchange of information between people and objects with extremely reduced latency and cost, high data rate, high capacity, and spectral and energy efficiency [1]. One of the research topics that attract the interest of both industry and academia with the coming of $5 \mathrm{G}$ systems is channel modelling. A variety of channel models has been proposed in the literature enabling design, performance evaluation of communication systems, and study of propagation characteristics [2]. However, these models are not compatible with $5 \mathrm{G}$ because they were designed for frequencies below $6 \mathrm{GHz}$. Thus, new channel models are needed for $5 \mathrm{G}$ systems at frequencies above $6 \mathrm{GHz}$ [3], and they should meet additional requirements which may include

(i) Supporting the massive explosion of connected devices and links, the world's demand of data is increasing, and researchers expect that number of connected devices can reach 50 billion by 2030 [4] which exceeds the population in the world that will be 8.5 billion people [5]

(ii) Being compatible with dual mobility of both transceivers in order to support scenarios such as device-to-device and vehicle-to-vehicle communication

(iii) Being practical computationally, in fact existent channel models are complex, especially Three-Dimensional (3D) channel models, which define a large number of parameters and are not practical in implementation

(iv) The new channel model must accommodate a higher frequency range in order to be suitable with the $5 \mathrm{G}$ system which can exceed $100 \mathrm{GHz}$ [6]

(v) Supporting high speed of devices, new channel models should be able to support the mobility speed of above $500 \mathrm{~km} / \mathrm{h}$ for some scenarios [7] 
Many studies have been conducted for frequencies above $6 \mathrm{GHz}$ in various international projects, such as Third Generation Partnership Project (3GPP), Wireless World INitiative NEw Radio (WINNER), COoperative in Science and Technology (COST), Mobile and wireless communications Enablers for the Twenty-twenty Information Society (METIS), and Millimetre-Wave Evolution for Backhaul and Access (MiWEBA), in order to develop channel models suitable for $5 \mathrm{G}$ scenarios, Multiple Input Multiple Output (MIMO) technology, and dual mobility systems.

Figure 1 summarizes those channel models, their year of occurrence, and their family history.

Channel estimation is crucial for design of wireless communication systems; more precisely, an estimated channel impulse response is important for channel equalization, demodulation, decoding, etc. In addition, it is essential for radio resource management and performance optimization. Moreover, accuracy of channel estimation is critical to decide whether wireless communication system is reliable or not.

MIMO channel estimation has been studied intensively in many research papers for different scenarios. The Alternating Direction Method of Multipliers (ADMM) [8] is an algorithm that solves convex optimization problems. This method has recently found wide application in a number of areas. In [9], a channel estimation algorithm for mmWave channels is proposed, and this algorithm is based on ADMM and provides the global optimum solution to the considered convex mmWave channel estimation problem with fast convergence properties. Multiple Signal Classification (MUSIC) algorithm has been proposed in [10], and it is one of the most known algorithms for spatial spectrum estimation. The basic idea of MUSIC algorithm is to conduct characteristic decomposition for the covariance matrix, resulting in a signal subspace orthogonal with a noise subspace; these two orthogonal subspaces are used to constitute a spectrum function. Spectral peaks are then detected to extract the direction of arrival signals. Another channel estimation and tracking method for correlated block-fading channels in massive MIMO wireless cellular systems is presented in [11]. A nonstationary channel estimation method based on the Extended Kalman Filter (EKF) is proposed in [12]. An advanced minimum mean square error (MMSE) channel estimation method for vehicle-to-vehicle (V2V) system has been proposed in [13] by applying the weighted sum using update matrix (WSUM) scheme. According to this study, existing estimation schemes present some limitations. In fact, many estimation algorithms are not applicable to communication scenarios such as V2V systems. In addition, existing channel estimation methods for $\mathrm{V} 2 \mathrm{~V}$ cannot effectively reduce intercarrier interference and present high-computation complexity [14]. These limitations motivate us to propose a new simplified method for parameter estimation of the Three-Dimensional model of Third Generation Partnership Project (3D-3GPP) channel model.

This paper focuses on the 3D-3GPP channel models, and it proposes a new method for parameter estimation of this model using Estimation of Signal Parameters via Rotational Invariant Techniques (ESPRIT).

The contributions of this paper are

(i) Proposing a new simplified method for parameter estimation of the 3D-3GPP channel model based on ESPRIT algorithm and providing the new expressions of Doppler frequencies and complex amplitudes of the channel

(ii) Verifying the simplified model with the original 3D3GPP model in terms of Doppler frequencies and complex amplitude

(iii) Evaluating the new model in terms computational time, computational complexity and normalized mean square error

The rest of this paper is organized as follows. The 3D3GPP channel model is presented and treated in Section 2. Section 3 describes the ESPRIT algorithm. The proposed estimation method is discussed in Section 4, and it is tested and evaluated in Section 5. Finally, conclusions are presented in Section 6.

\section{Third Generation Partnership Project Channel Models}

3GPP is the partnership between seven telecommunications standardization organization from Japan, North America China, Europe, India, and South Korea [3]. First, 3GPP has considered only Spatial Channel Model (SCM) which is a Two-Dimensional (2D) propagation model [15], introduced earlier in 2003 [16], and SCM was developed for the $2 \mathrm{GHz}$ frequency band with channel bandwidths up to $5 \mathrm{MHz}$ [17]. In 2005, its wideband extension referred to Spatial Channel Model extended (SCM-E) [18] was developed to support larger bandwidths, up to $100 \mathrm{MHz}$. This latter supports a frequency range of $2-6 \mathrm{GHz}$ as well. In 2017, the 3D model for 3GPP has been proposed in [19]. In fact, the existing $2 \mathrm{D}$ channel models do not consider the elevation channel characteristics which make them insufficient for some studies. The new version investigated for the first time for $\mathrm{V} 2 \mathrm{~V}$ communication channel in roadside scattering environment [15] and the impact of vehicle speed, traffic density, and angle of departure, angle of arrival, and other statistical performances on the V2V channel model is thoroughly discussed. In [19], the 3rd Generation Partnership Project (3GPP) has released a new $3 \mathrm{D}$ spatial channel model for frequencies from 0.5 to $100 \mathrm{GHz}$, and it is a 3D geometric stochastic model describing the scattering environment. The 3D-3GPP channel model specifies three propagation conditions: line-of-sight (LOS), non-line-of-sight (NLOS), and outdoor-to-indoor (O2I).

The 3D model of the 3GPP model is mainly a modified version of the SCM model; Figure 2 is a simplified representation of the 3D-3GPP channel model; equation (1) represents the $3 \mathrm{D}$ model of $\mathrm{V} 2 \mathrm{~V}$ communication system under MIMO conditions [20]: 


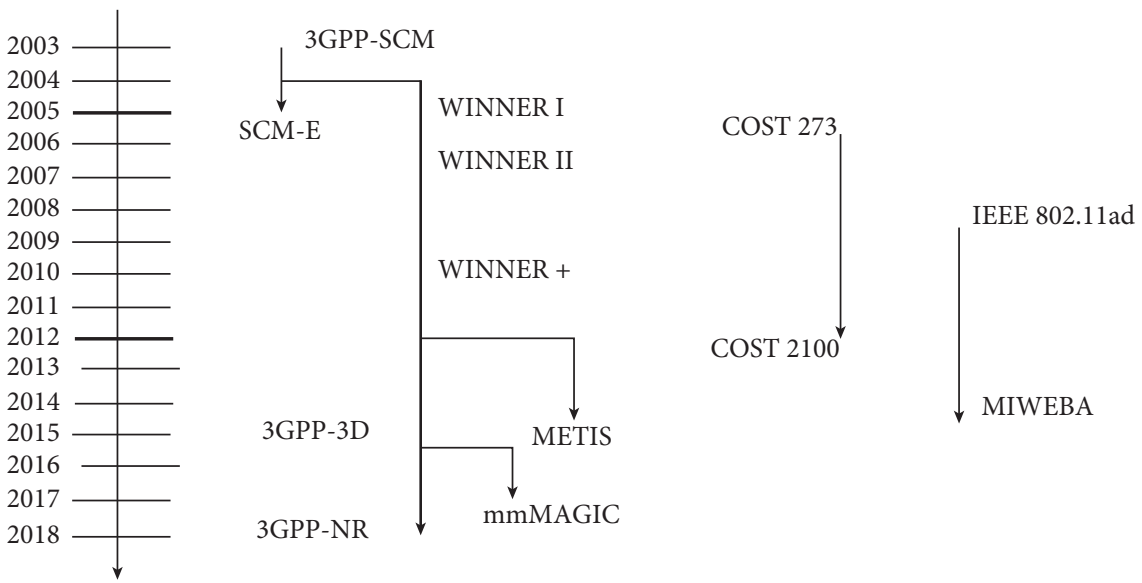

FIgURE 1: Standard channel models and their family history.

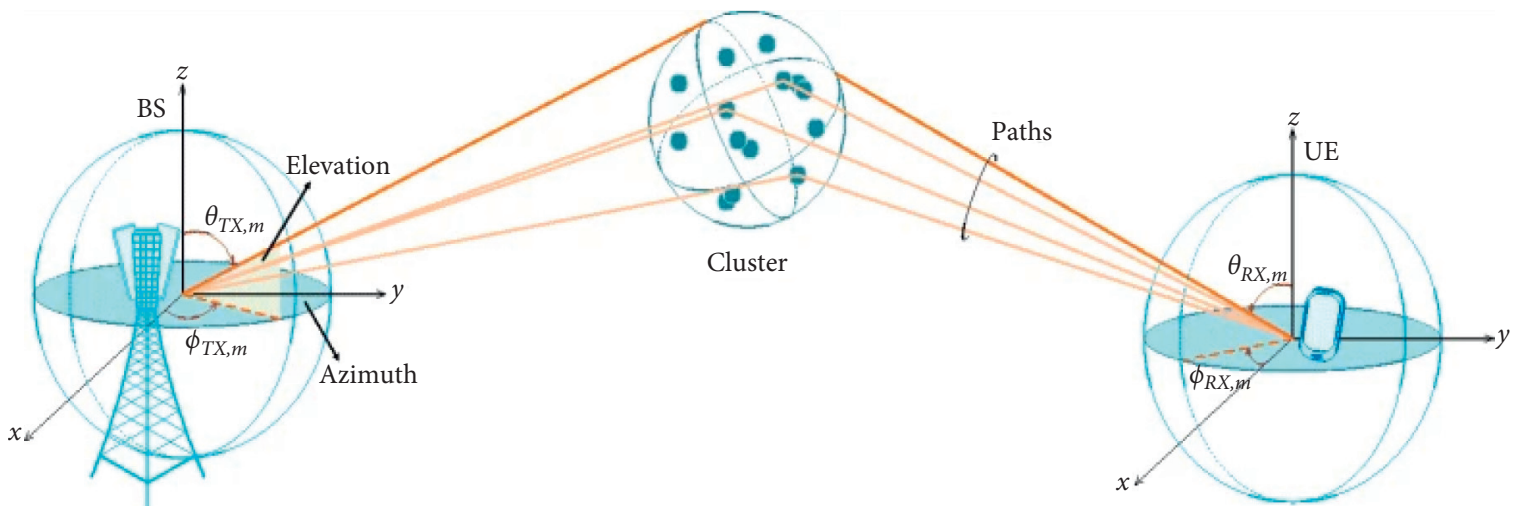

FIGURE 2: Simplified representation of the 3D-3GPP channel model [21].

$$
h(\tau)=\sqrt{P_{k}} \sum_{k=1}^{K}\left[\begin{array}{l}
F_{r x, u, V}\left(\theta_{k}\right) \\
F_{r x, u, H}\left(\theta_{k}\right)
\end{array}\right]^{T}\left[\begin{array}{cc}
e^{j \Phi_{k}^{v v}} & \sqrt{k_{k}} e^{j \phi_{k}^{v h}} \\
\sqrt{k_{k}} e^{j \Phi_{k}^{h v}} & e^{j \phi_{k}^{h h}}
\end{array}\right]\left[\begin{array}{c}
F_{t x, s, V}\left(\phi_{k}\right) \\
F_{t x, s, H}\left(\phi_{k}\right)
\end{array}\right] \times e^{\left(j 2 \pi \lambda^{-1} \overline{\theta_{k} \cdot \overline{\Gamma_{r x, u}}}\right)} \times e^{\left(j 2 \pi \lambda^{-1} \overline{\phi_{k}} \cdot \overline{\Gamma_{t x, s}}\right)} e^{\left(j w_{k} \tau\right)},
$$

where $P_{k}$ is the power of the $k^{\text {th }}$ path, $K$ is the number of paths, and $F_{r x, u, V}$ and $F_{r x, u, H}$ are the antenna element $u$ field patterns for vertical and horizontal polarizations, respectively. Furthermore, $\lambda$ is the wavelength of carrier frequency in meters, $\bar{\theta}_{k}$ is angle of departure (AoD) unit vector, $\bar{\phi}_{k}$ is angle of arrival (AoA) unit vector, $j$ is the square root of -1 , $\bar{\Gamma}_{t x, s}$ and $\bar{\Gamma}_{r x, u}$ are the location vectors of element $s$ and $u$, respectively, and $w_{k}$ is the effective radian Doppler frequency and can be represented by

$$
w_{k}=\frac{2 \pi}{\lambda}\left(v_{t} \cos \left(\theta_{k}\right)+v_{r} \cos \left(\phi_{k}\right)\right),
$$

where $v_{t}$ and $v_{r}$ are, respectively, transmitter and receiver velocities.

\section{Estimation of Signal Parameters via Rotational Invariant Techniques' Algorithm}

Several parameter estimation algorithms can be found in the literature. They are needed in order to obtain parameters for channel models, and they vary in their design criteria, statistical properties, computational complexity, and dimensionality [22]. ESPRIT algorithm is one of the most popular subspace methods in channel estimation [23].

Consider the system described in Figure 3, where $x(t)=$ $\left[x_{1}(t), x_{2}(t), \ldots, x_{P}(t)\right]$ is the input signal, $y(t)=\left[y_{1}(t), y_{2}(t), \ldots, y_{N}(t)\right]$ is the output signal. Based on ESPRIT algorithm, the output signal can be derived by the following expression:

$$
y(t)=A\left(\theta_{p}\right) x(t)+n(t)
$$

where $n(t)=\left[n_{1}(t), n_{2}(t), \ldots, n_{N}(t)\right]$ is the noise vector and $A\left(\theta_{p}\right)=\left[a_{j}^{i-1}\right]_{i=1, \ldots, N}$ is the steering matrix, $j=1, \ldots P$

where $N$ and $P$ are the dimensions of vectors $x(t)$ and $y(t)$, respectively. The goal is to estimate the arrival angles $\left\{\theta_{p}\right\}_{p=1}^{N}$ [24].

ESPRIT algorithm is composed by the following three steps: 


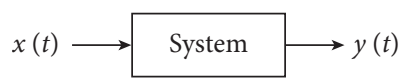

FIgURE 3: Simplified representation of a typical system.

Step 1: algorithm starts by building structured array steering matrix $A$ which is obtained as follows:

$$
A=\left[\begin{array}{cccc}
1 & 1 & \ldots & 1 \\
a_{1} & a_{2} & \ldots & a_{j} \\
\vdots & \vdots & \ddots & \vdots \\
a_{1}^{N-1} & a_{2}^{N-1} & \ldots & a_{P}^{N-1}
\end{array}\right]
$$

From $A$, two steering matrices $A_{1}$ and $A_{2}$ are derived as follows, which correspond to $A$ without first and last row, respectively:

$$
\begin{gathered}
A_{1}=\left[\begin{array}{cccc}
1 & 1 & \ldots & 1 \\
a_{1} & a_{2} & \ldots & a_{p} \\
\vdots & \vdots & \ddots & \vdots \\
a_{1}^{N-2} & a_{2}^{N-2} & \ldots & a_{P}^{N-2}
\end{array}\right], \\
A_{2}=\left[\begin{array}{cccc}
a_{1} & a_{2} & \ldots & a_{p} \\
a_{1}^{2} & a_{2}^{2} & \ldots & a_{p}^{2} \\
\vdots & \vdots & \ddots & \vdots \\
a_{1}^{N-1} & a_{2}^{N-1} & \ldots & a_{P}^{N-1}
\end{array}\right] .
\end{gathered}
$$

$A_{1}$ and $A_{2}$ can be related by the invariance equation:

$$
A_{1}=A_{2} \Phi
$$

where $\Phi$ is the diagonal matrix defined by

$$
\Phi=\left[\begin{array}{cccc}
a_{1} & 0 & \ldots & 0 \\
0 & a_{2} & \ldots & 0 \\
\vdots & \vdots & \ddots & \vdots \\
0 & 0 & \ldots & a_{p}
\end{array}\right]
$$

Step 2: if $A_{1}$ and $A_{2}$ are unknown and $E_{s}$ is the signal formed by subspace eigenvectors, $E_{s}$ and $A$ are related with the following equation:

$$
E_{s}=A T
$$

The ESPRIT algorithm begins by forming the invariance equation:

$$
E_{s 1}=E_{s 2} \psi
$$

where $E_{s 1}$ and $E_{s 2}$ are submatrices formed from $E_{s}$ similar to $A_{1}$ and $A_{2}$, and $\psi=T^{-1} \Phi T$.

Equation (10) is solved using the least square method:

$$
\psi=\left(E_{s 2}^{\dagger} E_{s 2}\right)^{-1}\left(E_{s 2}^{\dagger} E_{s 1}\right)
$$

where $\dagger$ refers to the Hermitian transpose.
Step 3: therefore, $\left\{\theta_{p}\right\}_{p=1}^{N}$ is estimated by the following equation:

$$
\theta_{p}=\sin ^{-1}\left[\frac{\arg \left(\lambda_{p}\right)}{2 \pi d}\right]
$$

where arg ( ) denotes the phase angle of the associated complex number on $(0 ; 2 \pi]$ and $\lambda_{p}$ are eigenvalues of $\psi$. Hence, from equation (3) and by following the 3 steps of ESPRIT algorithm, arrival angles $\left\{\theta_{p}\right\}_{p=1}^{N}$ are estimated in terms of eigenvalues of $\psi$.

\section{Proposed Parameter Estimation Method}

In order to reduce the complexity of channel prediction problem for the 3D-3GPP channel model, it will be considered as a sinusoidal parameter estimation problem. Based on equation (1), the complex amplitude of the $k^{\text {th }}$ path is denoted as $H_{L}$ which corresponds to a vector containing all the temporal samples; then,

$$
\beta_{k}=\alpha_{k} e^{j \phi_{k}}
$$

where

$$
\phi_{k}=2 \pi \lambda^{-1} \bar{\theta}_{k} \cdot \bar{\Gamma}_{r x, u}+2 \pi \lambda^{-1} \bar{\phi}_{k}
$$

and

$$
\alpha_{k}=\sqrt{P_{k}}\left[\begin{array}{c}
F_{r x, u, V}\left(\theta_{k}\right) \\
F_{r x, u, H}\left(\theta_{k}\right)
\end{array}\right]^{T}\left[\begin{array}{c}
e^{j \Phi_{k}^{v v}} \\
\sqrt{k_{k}} e^{j \Phi_{k}^{h v}} \\
\sqrt{k_{k}} e^{j \phi_{k}^{v h}} \\
e^{j \phi_{k}^{h h}}
\end{array}\right]\left[\begin{array}{c}
F_{t x, s, V}\left(\phi_{k}\right) \\
F_{t x, s, H}\left(\phi_{k}\right)
\end{array}\right] .
$$

Thus, equation (1) becomes

$$
h(t)=\sum_{k=1}^{K} \beta_{k} e^{j \omega_{k} t} .
$$

The proposed method for estimating the Doppler frequencies and complex amplitudes of the channel is based on the ESPRIT algorithm by following the same steps.

Assuming that $L$ samples of the channel are known, by transmitting known pilot sequences or from measurement, the sampling interval is $\Delta t$. A vector $H_{L}$ is given by

$$
H_{L}=\left[\begin{array}{c}
h(0) \\
h(\Delta t) \\
\cdot \\
\vdots \\
h((L-1) \Delta t)
\end{array}\right] .
$$

Using equation (16), $H_{L}$ can be written as

$$
H_{L}=F \beta \text {, }
$$

where 


$$
\begin{array}{r}
F=\left[\begin{array}{cccc}
1 & 1 & \ldots & 1 \\
f_{1} & f_{2} & \ldots & f_{k} \\
\vdots & \vdots & \ddots & \vdots \\
f_{1}^{L-1} & f_{2}^{L-1} & \cdots & f_{K}^{L-1}
\end{array}\right], \\
\beta=\left[\begin{array}{llll}
\beta_{1} & \beta_{2} & \cdots & \beta_{K}
\end{array}\right]^{T},
\end{array}
$$

where $f_{k}=e^{j w_{k} \Delta t}$

In order to determine both Doppler frequency and complex amplitude, this method uses the same steps as ESPRIT algorithm and applies to equation (18), where noise vector $n(t)$ is assumed to be zero.

Step 1: similar to $A_{1}$ and $A_{2}$ in equations (5) and (6), let $F_{1}$ and $F_{2}$ be $F$ without first and last row, respectively; then,

$$
\begin{aligned}
F_{1} & =\left[\begin{array}{cccc}
f_{1} & f_{2} & \ldots & f_{k} \\
f_{1}^{2} & f_{2}^{2} & \ldots & f_{k}^{2} \\
\vdots & \vdots & \ddots & \vdots \\
f_{1}^{L-1} & f_{2}^{L-1} & \ldots & f_{K}^{L-1}
\end{array}\right], \\
F_{2} & =\left[\begin{array}{cccc}
1 & 1 & \ldots & 1 \\
f_{1} & f_{2} & \ldots & f_{k} \\
\vdots & \vdots & \ddots & \vdots \\
f_{1}^{L-2} & f_{2}^{L-2} & \ldots & f_{K}^{L-2}
\end{array}\right] .
\end{aligned}
$$

As in equation (7), the following equation can be formed:

$$
F_{1}=\gamma F_{2},
$$

where $\gamma$ is $K \times K$ matrix expressed as

$$
\gamma=\left[\begin{array}{cccc}
f_{1} & 0 & \ldots & 0 \\
0 & f_{2} & \ldots & 0 \\
\vdots & \vdots & \ddots & \vdots \\
0 & 0 & \ldots & f_{k}
\end{array}\right]
$$

Step 2: the objective of this step is to determine the subspace matrix $E_{s}$; thus, Step 2 starts by computing the Hankel matrix $H$ and then obtains the temporal correlation matrix from it.

$F$ is unknown; let $H$ be a $P \times Q$ Hankel matrix in which each ascending skew-diagonal from left to right is constant:

$$
H=\left[\begin{array}{cccc}
h(0) & h(\Delta t) & \ldots & h((P-1) \Delta t) \\
h(\Delta t) & h(2 \Delta t) & \ldots & h(P \Delta t) \\
\vdots & \vdots & \ddots & \vdots \\
h((Q-1) \Delta t) & h(Q \Delta t) & \ldots & h((L-1) \Delta t)
\end{array}\right],
$$

where $P$ and $Q$ can be determined from the following two equations:

$$
P+Q=L+1
$$

and

$$
P=\frac{2}{3} L
$$

where the operator $x$ refers to the smallest integer greater than $x$.

Next, the temporal correlation matrix, $\widehat{R}$, is determined from $H$ and is expressed as

$$
\widehat{R}=\frac{H H^{\dagger}}{P} .
$$

Let signal subspace matrix $E_{s}$ be composed from the $k$ eigenvectors which correspond to the largest eigenvalues of $\widehat{R}, E_{s 1}$ and $E_{s 2}$ represent the matrix $E_{s}$ without the bottom and top rows respectively, and $\Phi$ be the subspace matrix and is a rotated version of $\gamma$; equation (27) is written as in equation (10):

$$
E_{s 2} \Phi=E_{s 1} \text {. }
$$

Like in equation (11), the solution of equation (27) is

$$
\Phi=\left(E_{s 2}^{\dagger} E_{s 2}\right)^{-1}\left(E_{s 2}^{\dagger} E_{s 1}\right) \text {. }
$$

Step 3: therefore, the effective radian Doppler frequencies are given by

$$
\widehat{w}_{k}=\frac{\arg \left(\lambda_{k}\right)}{\Delta t},
$$

where $\lambda_{k}$ is the $k^{\text {th }}$ eigenvalue of $\Phi$.

Like Doppler frequencies, complex amplitudes of different paths are computed via resolution of the linear equation (18) and by applying the same steps of ESPRIT algorithm, where $\beta$ is considered to be the unknown parameter, and matrix $F$ is supposed as

$$
\widehat{\beta}=\left(F^{\dagger} F+v I\right)^{-1} F^{\dagger} \widehat{h} .
$$

Using the estimated parameters, the $\mathrm{V} 2 \mathrm{~V}$ channel impulse response can be extrapolated by substituting the parameters in equations (29) and (30) into equation (16) for any desired time instant $\tau=L \Delta t,(L+1) \Delta t, \ldots \ldots$ :

$$
\widehat{h}(\tau)=\sum_{k=1}^{K} \widehat{\beta}_{k} e^{\widehat{j \omega}_{k} \tau} .
$$

\section{Results and Discussion}

In this section, simulation is used to evaluate and verify the simplified model with the original 3D-3GPP model in terms of Doppler frequencies, complex amplitude, computational time, computational complexity, and normalized mean square error. Parameters have been chosen according to the environment which is considered to be an urban area; vehicles' speeds are assumed to be equal around $50 \mathrm{~km} / \mathrm{h}$. Simulations were performed at $5.9 \mathrm{GHz}$, the operating 
frequency for $\mathrm{V} 2 \mathrm{~V}$ communication, the sampling interval is considered to be $1 \mathrm{~ms}$, and the number of samples is set to 100. Table 1 presents the other parameters used in the simulation. The analysis starts by comparing Doppler frequency and complex amplitude of both 3D-3GPP channel model and estimated model in terms of number of paths. Then, magnitude and phase of the two models are also compared in terms of time. In addition, the proposed model is evaluated in terms of computational time and computational complexity. Finally, it has been assessed according to normalized mean square error in three different environments: urban, suburban, and highway.

First, the Doppler frequencies of both the 3D-3GPP channel model and the estimated model are plotted in terms of number of paths $k$ in Figure 4. As expected the Doppler frequency vary according to number of paths and the estimated Doppler frequency $\widehat{w}_{k}$ match exactly the true Doppler frequency $w_{k}$ for different values of $k$. It is noticed that values of Doppler frequency are between -2000 and $2000 \mathrm{~Hz}$. The Doppler frequency can either be negative or positive depending on the direction of motion of the transceivers which is relative to the direction of arrival and direction of departure. This is the cause behind the change of Doppler frequency in the zigzag mode.

In the second part, the magnitude and phase of the complex amplitude for both the 3D-3GPP channel model and the estimated model are compared in Figure 5 in terms of number of paths. It is noticed that curves for the estimated values of the amplitude and phase of the complex amplitude fit very well the true model. Therefore, the proposed method is found to perform well in terms of similarity with the true model. The variation of magnitude and phase of complex amplitude between negative or positive values is also due to the variation of direction of motion of the transceivers.

In addition to the previous results, time-varying amplitude and phase of the 3D-3GPP model and estimated channel are also compared in Figure 6. The results show that $\widehat{h}$ is similar to $h$ for both amplitude and phase for any value of $k$, and the small difference in shape between curves is due to regularization parameter $v$ used during the computation of $h$. The change of magnitude and phase of $\widehat{h}$ and $h$ in the zigzag mode is caused by the change of direction of motion of the transceivers.

There are two essential ways for checking the performance of an algorithm: computational time and computational complexity. The computational time or running time corresponds to how long it takes for a computer to perform the channel estimation scheme. In contrast, computational complexity is a more abstract and general way of expressing performance, and it refers to the amount of resources required to run an algorithm. To be specific, it is a representation of the asymptotic behavior of the model. In this study, the proposed model is evaluated in terms of computational time which represents the time during in which the channel model is computed. Figure 7 displays the variation of the computational time in seconds versus the number of paths for both of the true and the estimated model using MUSIC algorithm. From the obtained curves, it can be observed that the true model's computational time is higher than the estimated one and even starts to increase
TABLE 1: Simulation parameters.

\begin{tabular}{lc}
\hline Parameters & Parameter values \\
\hline Transmitter velocity & $50 \mathrm{~km} / \mathrm{h}$ \\
Receiver velocity & $50 \mathrm{~km} / \mathrm{h}$ \\
Angle of departure & Uniform over $[-\pi, \pi]$ \\
Angle of arrival & Uniform over $[-\pi, \pi]$ \\
Amplitude & Normalized over $(0,1)$ \\
Phase & Uniform over $[0,2 \pi]$ \\
Number of paths: $K$ & $5-30$ \\
\hline
\end{tabular}

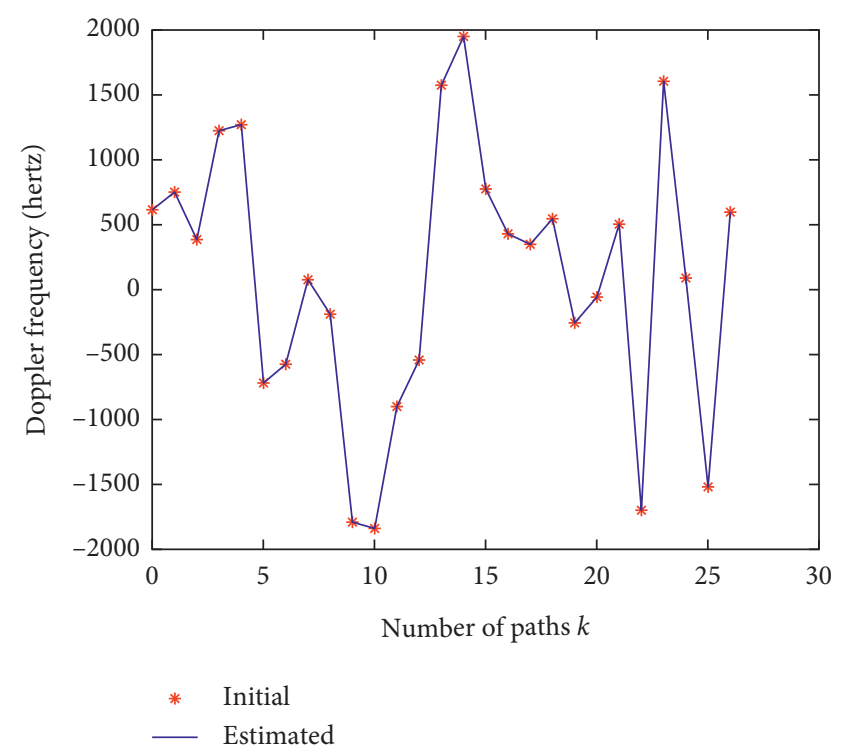

FIGURE 4: Doppler frequencies for 3D-3GPP and estimated model.

exponentially when the number of paths increases. On the contrary, the computational time for the proposed estimated method increases slowly as the number of paths increases and looks like it has a stable behavior; thus, it is clear that proposed model is much faster and more efficient in estimating the 3D-3GPP model in terms of computational time. Even though the estimated model using MUSIC algorithm has a stable behavior and performs better than the true model, the proposed model presents a better effectiveness.

To describe computational complexity of a function Big $O$ notation is used. According to equation (1) and Figure 7, the 3D-3GPP channel model presents a computational complexity having the behavior of exponential function, namely, $O\left(e^{n}\right)$. Whereas, according to equation (31) and Figure 7, the estimated model presents a behavior of $O(1)$, which proves the effectiveness of the proposed model in terms of computational complexity.

Finally, the proposed estimated method is also evaluated in terms of the Normalized Mean Square Error (NMSE) parameter which is defined as [25]

$$
\mathrm{NMSE}=\frac{E\left[|\widehat{h}(\tau)-h(\tau)|^{2}\right]}{E\left[|h(\tau)|^{2}\right]},
$$

where $E[$.$] refers to the expectation operator. Simulations$ were performed at $5.9 \mathrm{GHz}$ frequency; number of paths is set 


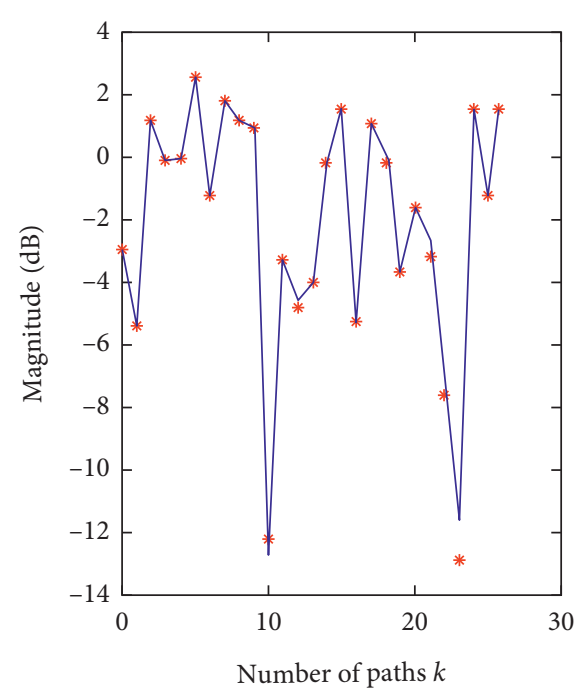

* Initial

- Estimated

(a)

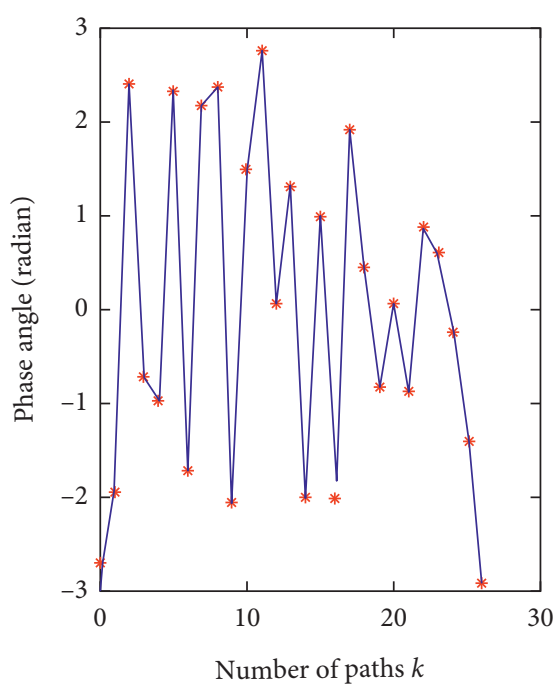

* Initial

_ Estimated

(b)

Figure 5: (a) Magnitude and (b) phase of the complex amplitude $\beta_{k}$ and $\widehat{\beta}_{k}$.

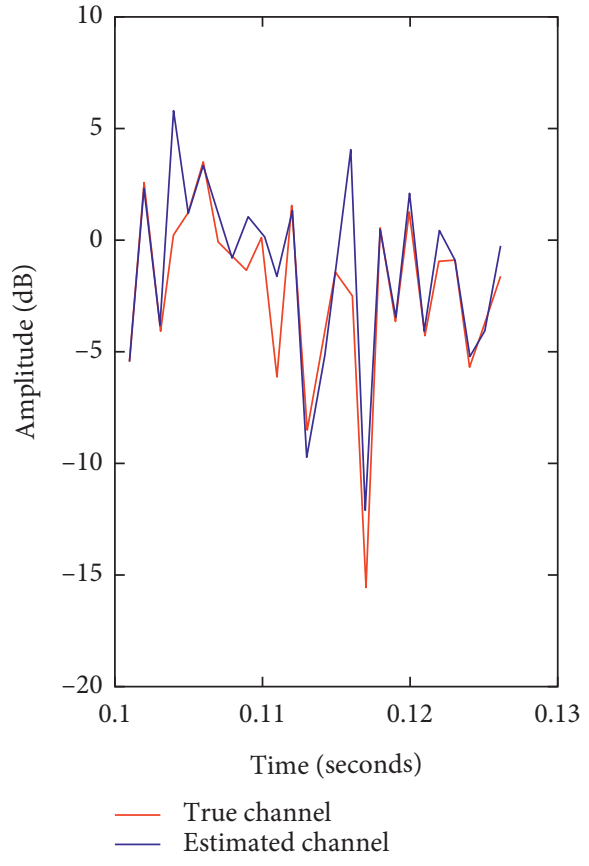

(a)

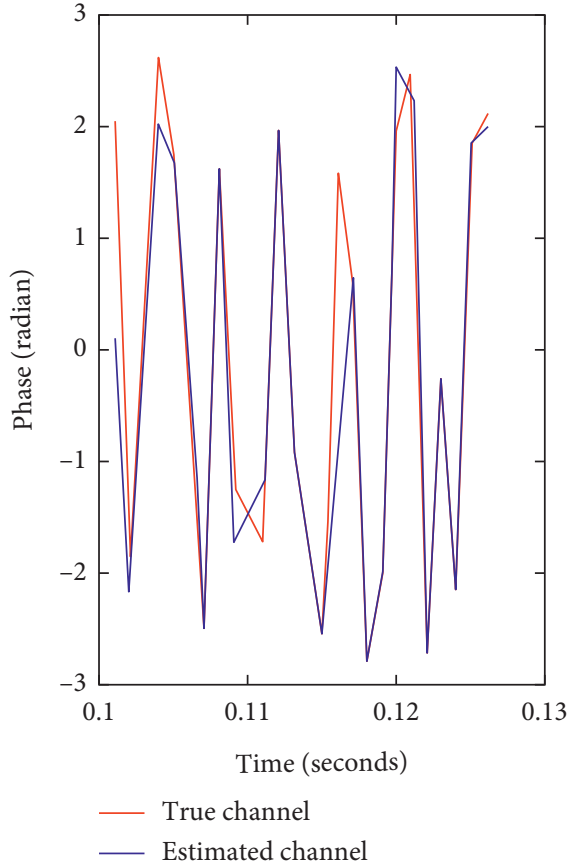

(b)

Figure 6: (a) Magnitude and (b) phase of $\hat{h}$ and $h$.

to 8,16 , and 32 in order to see the influence of number of paths on the variation of NMSE. Three different environments were considered: urban, suburban, and highway, and each environment is characterized by the velocity of transceivers, as it is mentioned in Table 2. Figures 8-10 present the variation of NMSE in terms of prediction horizon for three different environments. It can be seen from the obtained curves that NMSE is less than $-20 \mathrm{~dB}$ for all chosen environments and for all prediction horizon; thus, the estimated and true channel model are converging to each 


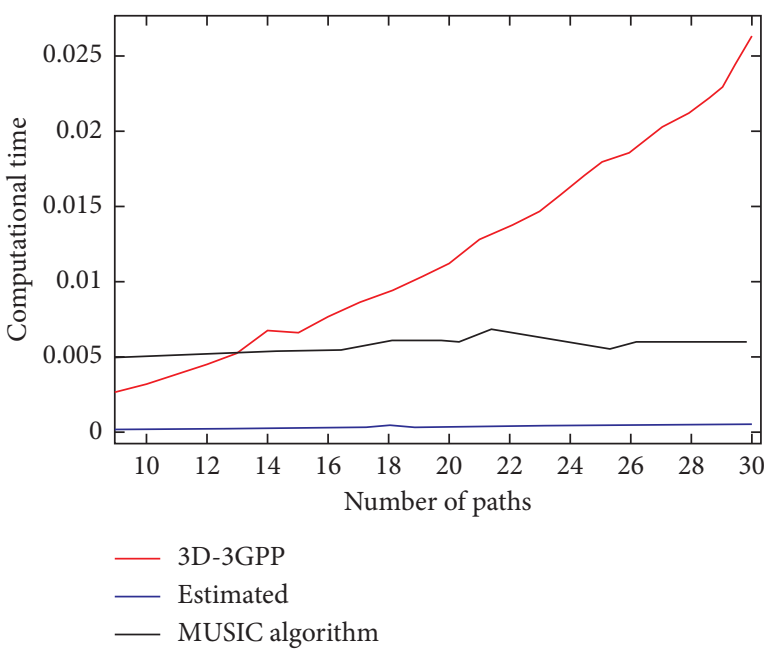

Figure 7: Computational time vs. number of paths of $\widehat{h}$ and $h$ : $v_{t}=v_{r}=50 \mathrm{~km} / \mathrm{h}$ and $\tau=20 \mathrm{~ms}$.

TABLE 2: Velocities characterizing environments.

\begin{tabular}{lr}
\hline Environment & Velocity \\
\hline Urban & $50 \mathrm{~km} / \mathrm{h}$ \\
Suburban & $70 \mathrm{~km} / \mathrm{h}$ \\
Highway & $110 \mathrm{~km} / \mathrm{h}$ \\
\hline
\end{tabular}

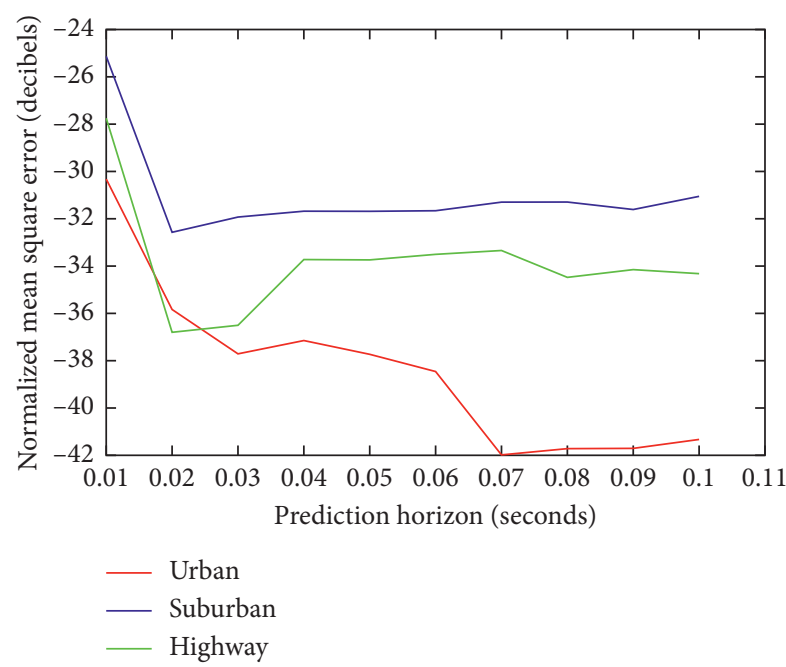

FIGURE 8: NMSE vs. prediction horizon for different urban, suburban, and highway environments with $k=8, L=100$, and $\Delta t=1 \mathrm{~ms}$.

other and relative error is of the order of $10^{-1}$ percent which is very good and can confirm the effectiveness of the proposed method. In addition, NMSE presents the minimum shape for urban environment in comparison under three different environmental conditions. Therefore, the proposed estimated model performs better with such environment.

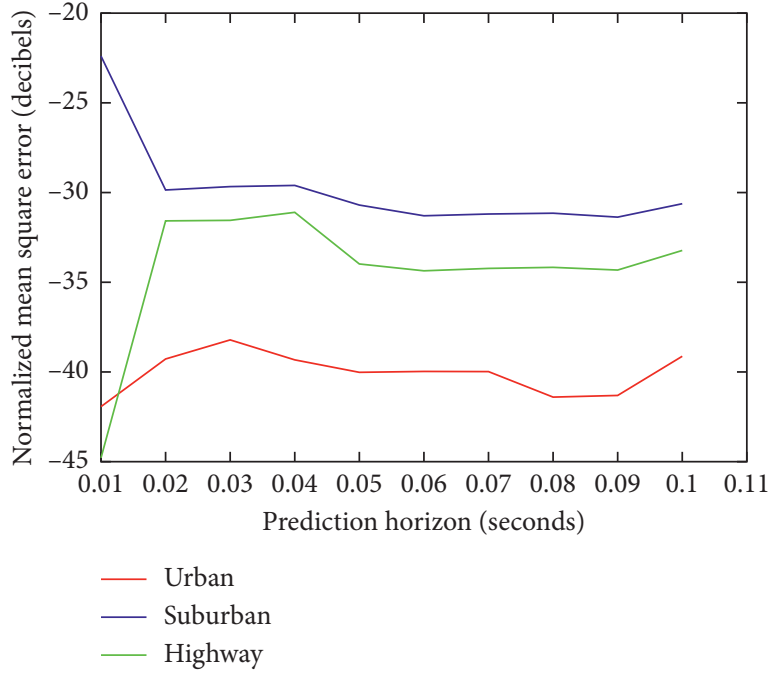

FIgURE 9: NMSE vs. prediction horizon for different urban, suburban, and highway environments with $k=16, L=100$, and $\Delta t=1 \mathrm{~ms}$.

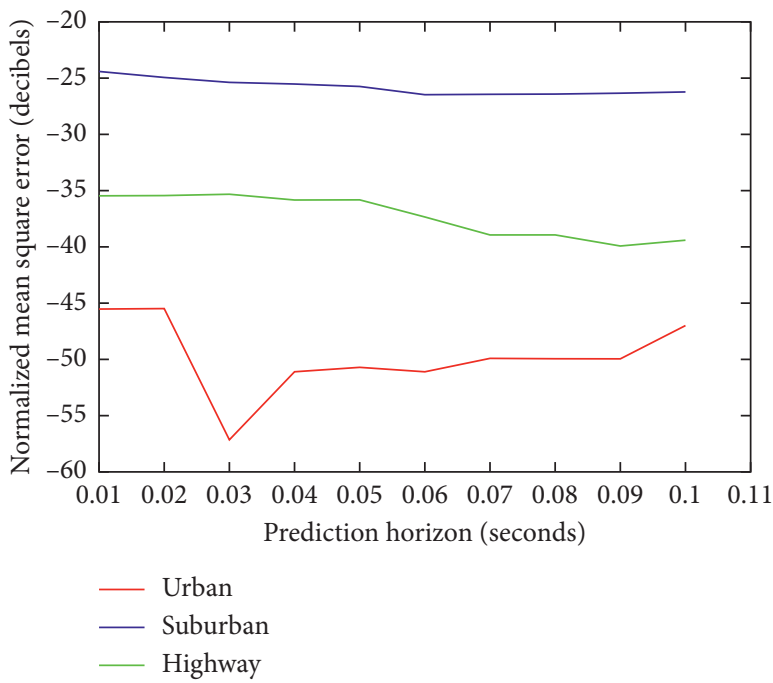

FIgURE 10: NMSE vs. prediction horizon for different urban, suburban, and highway environments with $k=32, L=100$, and $\Delta t=1 \mathrm{~ms}$.

\section{Conclusions}

Nowadays, channel modelling is attracting the interest of both industry and academia more, which resulted in the increased number of proposed channel models. However, most of these models, especially three-dimensional ones, are complex and take a long time to be resolved. This paper proposes a fast and simplified 3D-3GPP channel models using ESPRIT algorithm. Evaluation was performed at $5.9 \mathrm{GHz}$ frequency; it is found that the proposed model is in close agreement with the true model; in fact, estimated parameters such as Doppler frequency and complex amplitude match exactly the true model. In addition, the new proposed method is easier to use and requires much less 
computational time. It has also been shown that the evaluated normalized mean square error is generally less than $-25 \mathrm{~dB}$ which corresponds to very low relative errors. Therefore, it can be concluded that the proposed method improves the parameter estimation of the 3D-3GPP model when simulating a V2V communication channel. The proposed model can be used as a guide for design of wireless communication systems, radio resource management, and performance optimization. Furthermore, it helps to decide whether wireless communication system is reliable or not. The major limitations of this paper reside in the absence of measurements. Therefore, future research efforts may be devoted to conducting measurement campaigns cooperating with other universities or industries.

\section{Data Availability}

The data used to support the findings of the study are included within the article.

\section{Conflicts of Interest}

The authors declare that they have no conflicts of interest.

\section{References}

[1] J. G. Andrews, S. Buzzi, W. Choi et al., "What will 5G be?" IEEE Journal on Selected Areas in Communications, vol. 32, no. 6, pp. 1065-1082, 2014.

[2] H. Jiang and G. Gui, Channel Modeling in 5G Wireless Communication Systems, Springer, Berlin, Germany, 2020.

[3] A. Morgado, K. M. S. Huq, S. Mumtaz, and J. Rodriguez, "A survey of 5G technologies: regulatory, standardization and industrial perspectives," Digital Communications and Networks, vol. 4, no. 2, pp. 87-97, 2018.

[4] J. Pedro Tomás, "Global IoT connections to reach 50 billion by 2030: study," 2019, https://enterpriseiotinsights.com/ 20190520/internet-of-things/global-iot-connections-reach50-million-2030-study.

[5] United Nations Report, UN Projects World Population to Reach 8.5 Billion by 2030, Driven by Growth in Developing Countries, United Nations Report, New York, NY, USA, 2015.

[6] K. Haneda, J. Zhang, L. Tan et al., "5G 3GPP-like channel models for outdoor urban microcellular and macrocellular environments," in Proceedings of the 2016 IEEE 83rd Vehicular Technology Conference (VTC Spring), Nanjing China, May 2016.

[7] P. Fan, J. Zhao, and I. Chih-Lin, "5G high mobility wireless communications: challenges and solutions," China Communications, vol. 13, no. S2, pp. 1-13, 2016.

[8] S. Boyd, N. Parikh, E. Chu, B. Peleato, and J. Eckstein, "Distributed optimization and statistical learning via the alternating direction method of multipliers," Foundations and Trends in Machine Learning, vol. 3, no. 1, pp. 1-122, 2011.

[9] E. Vlachos, G. C. Alexandropoulos, and J. Thompson, "Massive MIMO channel estimation for millimeter wave systems via matrix completion," IEEE Signal Processing Letters, vol. 25, no. 11, pp. 1675-1679, 2018.

[10] T. Pham and M. F. Fong, "Real-time implementation of MUSIC for wideband acoustic detection and tracking," in Proceedings of the SPIE AeroSense'97: Automatic Target Recognition VII, pp. 250-256, Orlando, FL, USA, June 1997.
[11] D. Suresh and A. K. Singh, "Channel estimation and channel tracking for correlated block-fading channels in massive MIMO systems," Digital Communications and Networks, vol. 4, no. 2, pp. 138-147, 2018.

[12] Y. Liao, X. Shen, X. Dai, M. Xhao, D. Li, and X. Zhou, "EKFbased joint channel estimation and decoding design for nonstationary OFDM channel," in Proceedings of the GLOBECOM 2017-2017 IEEE Global Communications Conference, pp. 1-6, IEEE, Singapore, December 2017.

[13] D. Jang and K. Ko, "Advanced channel estimation method for IEEE 802.11p/WAVE system," International Journal of Contents, vol. 15, no. 4, pp. 27-35, 2019.

[14] Y. Liao, X. Shen, G. Sun, X. Dai, and S. Wan, "EKF/UKFbased channel estimation for robust and reliable communications in V2V and IIoT," EURASIP Journal on Wireless Communications and Networking, vol. 2019, no. 1, p. 144, 2019.

[15] X. Chen, Y. Fang, W. Xiang, and L. Zhou, "Research on spatial channel model for vehicle-to-vehicle communication channel in roadside scattering environment," Hindawi International Journal of Antennas and Propagation, vol. 2017, Article ID 3098198, 12 pages, 2017.

[16] I. Xirouchakis, "Spatial channel model for multiple input multiple output (MIMO) simulations," Global Reporting Initiative, Amsterdam, Netherlands, 3GPP TR 25.996, 2003.

[17] J. M. Meredith, "Spatial channel model for multiple input multiple output (MIMO) simulations," Global Reporting Initiative, Amsterdam, Netherlands, 3GPP TR 25.996, 2012.

[18] D. S. Baum, J. Hansen, J. Salo, G. D. Galdo, M. Milojevic, and P. Kyösti, "An interim channel model for beyond-3G systems: extending the 3GPP spatial channel model (SCM)," in Proceedings of the 2005 IEEE 61st Vehicular Technology Conference, vol. 5, pp. 3132-3136, Stockholm, Sweden, May 2005.

[19] J. M. Meredith and J. Krause, "Study on channel model for frequencies from 0.5 to $100 \mathrm{GHz}$," Global Reporting Initiative, Amsterdam, Netherlands, 3GPP TR 38.901, 2017.

[20] C. Arunachalaperumal, S. Dhilipkumar, and G. Abija, "Enhanced 3D MIMO channel for urban macro environment," International Journal of Pure and Applied Mathematics, Special Issue, vol. 118, no. 10, pp. 259-269, 2018.

[21] F. Ademaj, M. Taranetz, and M. Rupp, "3GPP 3D MIMO channel model: a holistic implementation guideline for open source simulation tools," EURASIP Journal on Wireless Communications and Networking, vol. 2016, no. 1, p. 14, 2016.

[22] Y. Yang, J. Xu, G. Shi, and C.-X. Wang, Guang Shi, 5G Wireless Systems: Simulation and Evaluation Techniques, Springer, Berlin, Germany, 2018.

[23] R. O. Adeogun, Channel Prediction for Mobile MIMO Wireless Communication Systems, Victoria University of Wellington, Wellington, New Zealand, 2015.

[24] C. R. Dongarsane, A. N. Jadhav, and S. M. Hirikude, "Performance analysis of ESPRIT algorithm for smart antenna system," International Journal of Communication Networks and Security, vol. 1, no. 3, p. 7, 2012.

[25] R. O. Adeogun, P. D. Teal, and P. A. Dmochowski, "Extrapolation of MIMO mobile-to-mobile wireless channels using parametric-model-based prediction," IEEE Transactions on Vehicular Technology, vol. 64, no. 10, pp. 4487-4498, 2015. 